\title{
Task-driven PCA-based design optimization of wearable cutaneous devices
}

\author{
Claudio Pacchierotti ${ }^{1}$, Eric M. Young ${ }^{2}$, and Katherine J. Kuchenbecker ${ }^{2,3}$
}

\begin{abstract}
Small size and low weight are critical requirements for wearable and portable haptic interfaces, making it essential to work toward the optimization of their sensing and actuation systems. This paper presents a new approach for task-driven design optimization of fingertip cutaneous haptic devices. Given one (or more) target tactile interactions to render and a cutaneous device to optimize, we evaluate the minimum number and best configuration of the device's actuators to minimize the estimated haptic rendering error. First, we calculate the motion needed for the original cutaneous device to render the considered target interaction. Then, we run a principal component analysis (PCA) to search for possible couplings between the original motor inputs, looking also for the best way to reconfigure them. If some couplings exist, we can re-design our cutaneous device with fewer motors, optimally configured to render the target tactile sensation. The proposed approach is quite general and can be applied to different tactile sensors and cutaneous devices. We validated it using a BioTac tactile sensor and custom plate-based 3-DoF and 6-DoF fingertip cutaneous devices, considering six representative target tactile interactions. The algorithm was able to find couplings between each device's motor inputs, proving it to be a viable approach to optimize the design of wearable and portable cutaneous devices. Finally, we present two examples of optimized designs for our 3-DoF fingertip cutaneous device.
\end{abstract}

Index Terms-Haptics and Haptic Interfaces, Mechanism Design, Wearable Robots, Human-Centered Robotics

\section{INTRODUCTION}

C UTANEOUS feedback is known to play a paramount role in enhancing the performance and effectiveness of teleoperated, virtual reality, and augmented reality immersive systems [1], [2]. Moreover, providing cutaneous-only cues in bilateral teleoperation has been proven to convey rich information to the human operator while not affecting the stability and safety of the control loop [3]. Cutaneous feedback technologies have also attracted the interest of leading IT companies, mostly to provide effective ungrounded haptic feedback through wearable devices. For example, the Apple Watch includes a linear actuator for delivering realistic vibrations, and Microsoft has presented a set of mechanically actuated handheld controllers [4].

Manuscript received: September 10, 2017; Revised January 4, 2018; Accepted February 12, 2018.

This paper was recommended for publication by Editor Yasuyoshi Yokokohji upon evaluation of the Associate Editor and Reviewers' comments. This research has received funding from Rennes Métropole under the "Allocation d'Installation Scientifique (AIS) 2017" programme.

${ }^{1}$ C. Pacchierotti is with CNRS, Univ Rennes, Inria, IRISA, Rennes, France claudio.pacchierotti@irisa.fr.

${ }^{2}$ Eric M. Young and Katherine J. Kuchenbecker are with the Mechanical Engineering and Applied Mechanics Department, University of Pennsylvania Philadelphia, USA. yoericeseas . upenn. edu.

${ }^{3}$ Katherine J. Kuchenbecker is also with the Haptic Intelligence Department, Max Planck Institute for Intelligent Systems, Stuttgart, Germany kjk@is.mpg.de.

Digital Object Identifier (DOI): see top of this page.
Wearable haptic devices are often designed for the fingertip since it is one of the most sensitive parts of the body and the one most often used for grasping, manipulation, and probing the environment. As reviewed in [5], popular techniques to provide cutaneous feedback to the fingertips are shearing belts and tactors, pneumatic jets and balloons, pin arrays, vibrotactile motors, and moving platforms, which can orient and/or translate on the finger pulp. Since a top design requirement for wearable haptic devices is to minimize size and weight, it is essential to understand which types of haptic stimuli are salient for the target application and optimize the design of the wearable device accordingly (e.g., type of end effector, bandwidth, number and placement of the actuators).

This paper presents a novel task-driven algorithm for the design optimization of fingertip cutaneous haptic interfaces. Given one or more target tactile interactions to render and a cutaneous device to optimize, the algorithm computes the minimum subset and optimal configuration of the device's actuators to minimize the estimated haptic rendering error. First, the target tactile interaction is recorded by a biomimetic sensor. Then, the data-driven algorithm of [6], [7] is used to calculate the motor commands that can render the considered target interaction over time at the operator's skin. Finally, principal component analysis (PCA) is used to look for possible couplings/synergies between the original motor inputs and indicate the best way to reconfigure the motors to minimize the estimated haptic rendering error. This general approach can be applied to different tactile sensors (used to register the target tactile sensation) and cutaneous devices. In this paper, we validate the algorithm using a BioTac tactile sensor and two custom platform-based fingertip cutaneous devices, shown in Figs. 1 and 2, respectively.

\section{Haptic Sensing And Actuation}

The BioTac tactile sensor mimics the physical properties and sensory capabilities of the human fingertip [8], [9], by combining three complementary sensing systems: deformation, internal fluid pressure, and temperature. Contact interactions deform the elastic skin and displace the underlying conductive fluid, changing the impedances between ground and 19 electrodes distributed over the surface of the rigid core. The DC pressure of the conductive fluid is measured by a hydroacoustic pressure sensor, which also detects the AC pressure changes caused by transient contacts and textures.

The first haptic device we considered is a 3-DoF fingertip cutaneous device, presented in [3], [6] and shown in Fig. 1. It is composed of a static platform that houses three servo motors above the user's fingernail and a mobile platform that applies the requested stimuli to the fingertip. Three 


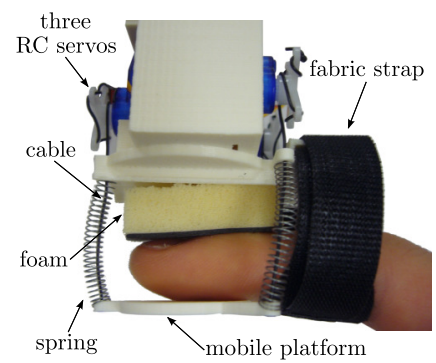

(a) 3-DoF fingertip device.

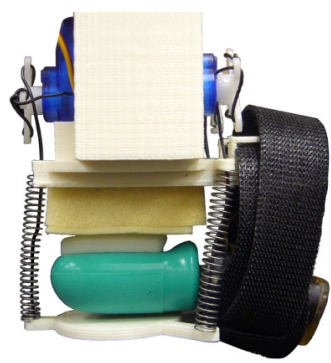

(b) Data collection setup.
Fig. 1. The 3-DoF device is composed of a static platform that houses three servo motors and a mobile platform that applies tactile stimuli to the fingertip [3], [6]. By controlling the lengths of the cables connecting the two platforms, the motors can orient and translate the platform in three-dimensional space.

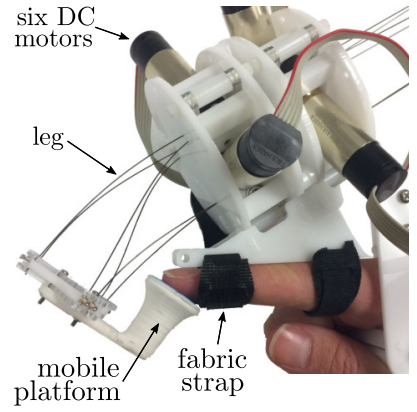

(a) 6-DoF fingertip device.

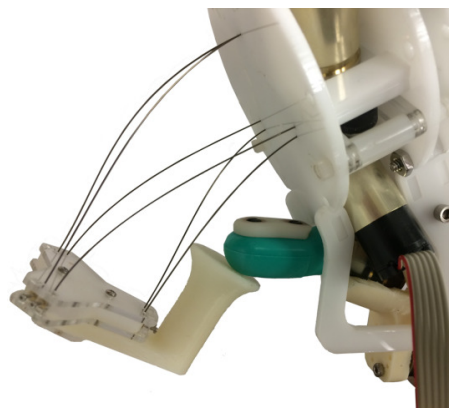

(b) Data collection setup.
Fig. 2. The 6-DoF device is a parallel continuum manipulator, consisting of six parallel, compliant legs passing through a fixed base platform [10]. The end-effector's pose can be controlled by independently adjusting the six leg lengths via six motors located above the finger.

cables connect the two platforms, and springs around the cables keep the mobile platform in a reference configuration, away from the fingertip, when not actuated. By controlling the cable lengths, the motors can orient and translate the mobile platform in three-dimensional space. The second haptic device we considered is a 6-DoF fingertip cutaneous device, preliminarily presented in [10] and shown in Fig. 2. It is a parallel continuum manipulator, consisting of six parallel, compliant legs that pass through a fixed base platform and attach to the distal platform. The end-effector's pose can be controlled by independently adjusting the six leg lengths (the wire between the two platforms) via six motors in the base platform, allowing the mobile portion of the robot to remain lightweight. A video showing the two devices is available as supplemental material.

Given the 19 electrode impedances distributed over the BioTac's surface and the DC pressure of the conductive fluid, it is not trivial to control the orientation and position of the mobile platforms to recreate the contact deformations applied to the sensor [6], [7], [11]. In this respect, Pacchierotti et al. [6], [7] have recently presented a data-driven model-free approach to map contact deformations sensed by the BioTac to input commands for the cutaneous device's motors. We summarize this approach in the next section.

\section{MAPping Sensed Data to Motor Commands}

Our goal is to enable the user to perceive, through the fingertip cutaneous devices, the deformations experienced by the BioTac as it interacts with the remote environment. In other words, we aim to find a good many-to-few mapping between the rich sensory information measured by the BioTac and the limited actuation capabilities of each fingertip cutaneous device. This algorithm was presented and evaluated in [6], [7] for the 3 DoF cutaneous device of Fig. 1. This Section summarizes that approach and generalizes it for an $N$-DoF cutaneous device.

The BioTac registers the electrode data and DC pressure with a precision of 12 bits at $100 \mathrm{~Hz}$. Let $s(j) \in \mathbb{S}=$ $\left\{\left(s_{1}(j), \ldots, s_{20}(j)\right) \in \mathbb{Z}^{20}: 0 \leq s_{i}(j) \leq 4095\right\}$ be a vector containing the electrode and DC pressure values sensed at instant $j$. In contrast, our two cutaneous feedback devices use three and six motors. Let $\boldsymbol{m}(\boldsymbol{j}) \in \mathbb{M}=$ $\left\{\left(m_{1}(j), m_{2}(j), \ldots, m_{N}(j)\right) \in \mathbb{R}^{N}\right\}$ be a vector containing the commanded inputs for the $N$ motors of the considered cutaneous device at instant $j$. For example, our 3-DoF cutaneous device has $N=3$ position-controlled servo motors, and therefore $\boldsymbol{m}(\boldsymbol{j})$ will contain the three commanded motor angles. On the other hand, our 6-DoF cutaneous device has $N=6$ current-controlled DC motors, which regulate the length of six legs; in this case, $\boldsymbol{m}(\boldsymbol{j})$ will contain the six commanded leg lengths. To simplify the notation, the sampling time index $j$ will be omitted from now on.

The objective of this algorithm is to map a given BioTac sensation, defined by $s$, to a congruent configuration of the mobile platform, defined by motor inputs $\boldsymbol{m}$. To address this problem, we placed the BioTac inside the cutaneous device and tested how the motion of the mobile platform affects the fingertip deformation readings.

\section{A. Data collection}

We ran one data collection per device. As shown in Figs. 1b and $2 \mathrm{~b}$, the BioTac was placed inside each cutaneous device in the same way a human user would wear the devices (compare with Figs. 1a and 2a). We then moved the mobile platform to a wide range of configurations and recorded the effect of each of these configurations on the BioTac, saving both the commanded motor inputs $\boldsymbol{m}_{*}$ (i.e., motor angles for the 3-DoF device and leg lengths for the 6-DoF device) and the resulting effect on the tactile sensor's electrodes and DC pressure $s_{*}$ [6]. Deciding how many and which platform configurations to test during data collection is an intriguing and challenging problem, which is, however, out of the scope of this paper; this important issue will be tackled in the future (see Sec. VI). In this work, we registered the effect on the BioTac of 166375 and 4033 platform configurations for the 3-DoF and 6-DoF devices, respectively. Data collection lasted approximately 47 hours for our 3-DoF device and approximately 1 hour for our 6-DoF device. Although here we considered motor angles and leg lengths as the motor inputs $\boldsymbol{m}_{*}$ for the two devices, one could also use other descriptors of the end-effector's state, such as the platform position and/or orientation.

The collected data defines a look-up table of the fingertip deformations caused by motion of the mobile platform, and it enables us to evaluate the mapping function

$$
\mu: \mathbb{S}_{*} \rightarrow \mathbb{M}_{*}, \quad \mu\left(s_{*}\right)=\boldsymbol{m}_{*},
$$

which links the BioTac's electrode and DC pressure readings to the corresponding tested motor inputs. Each device has a 
different look-up table that depends on its kinematic structure and actuation capabilities. The proposed approach can be applied to any similar cutaneous device and tactile sensor.

\section{B. From the BioTac to the motors}

After the data collection, we need a function able to map a generic sensed point $s \in \mathbb{S}$ to one in our mapping function's domain $\mathbb{S}_{*}$. We address this problem by looking for the $n$-points in our domain closest to the sensed one, thus defining

$$
\nu: \mathbb{S} \rightarrow \mathbb{S}_{*}^{n}, \quad \nu(s)=\left[\begin{array}{c}
s_{*, 1} \\
\vdots \\
s_{*, n}
\end{array}\right]=\hat{s}_{*},
$$

as the function that maps a generic point $s \in \mathbb{S}$, sensed by the BioTac, to the $n$ closest ones in $\mathbb{S}_{*}$. We use $n=8$ because we previously found that retrieving the eight closest points provides a good trade-off between tactile output performance and computational load [6], [7]. We can now map the $n$ points retrieved by $\nu(\cdot)$ to their corresponding motor input $N$-tuple from data collection,

$$
\mu: \mathbb{S}_{*}^{n} \rightarrow \mathbb{M}_{*}^{n}, \mu\left(\widehat{s}_{*}\right)=\mu\left(\left[\begin{array}{c}
s_{*, 1} \\
\vdots \\
s_{*, n}
\end{array}\right]\right)=\left[\begin{array}{c}
\boldsymbol{m}_{*, \mathbf{1}} \\
\vdots \\
\boldsymbol{m}_{*, n}
\end{array}\right]=\widehat{\boldsymbol{m}}_{*},
$$

Finally, we can average $\widehat{m}_{*} \in \mathbb{M}_{*}^{n}$ to a single $N$-tuple for the motors as

$$
\varphi: \mathbb{M}_{*}^{n} \rightarrow \mathbb{M}, \quad \varphi\left(\widehat{\boldsymbol{m}}_{*}\right)=\overline{\boldsymbol{m}},
$$

considering a mean that weights motor $N$-tuples according to the inverse squared distance between the corresponding point in $\mathbb{S}_{*}$ and the one sensed by the BioTac [6]. Vector $\overline{\boldsymbol{m}} \in \mathbb{M}$ is our final command for the device at this time step.

This rendering approach has been already proven successful for the off-line rendering of pre-recorded tactile interactions [6], the on-line rendering of tactile sensations recorded during robotassisted palpation with a da Vinci surgical robot [7], and the comparison of fingertip cutaneous displays during pinching palpation with a da Vinci surgical robot [12].

\section{DATA REDUCTION AND TASK DIMENSIONALITY}

This paper presents a novel approach for the task-driven design of cutaneous haptic devices. It aims at finding the best number and configuration of motors a cutaneous device needs to effectively render one or more target tactile interactions. The proposed approach can be useful in the fields of wearable haptics, immersive reality, teleoperation, rehabilitation, and in all the related fields and applications where it is important to employ small and lightweight haptic devices. For example, referring to the classification of Exploratory Procedures (EPs) described by Lederman and Klatzky [13], if our target tactile interaction includes solely the "pressure" EP, we may not need more than one degree of freedom to properly render the target interaction to the human operator. In this case, our cutaneous device may consist of only one motor, e.g., that moves a platform toward/away from the fingertip (such as in [14]). Similarly, if our target tactile interaction is comprised of a combination of "pressure" and "lateral motion" EPs, we may not need more than three degrees of freedom to properly render the target interaction to the human operator. In this case, our cutaneous device may need only three motors, e.g., that move a platform toward/away from the fingertip as well as tangentially with respect to its volar surface (such as in [1], [15]). Our algorithm aims at automatically finding these pieces of information - number and configuration of motors needed - starting from a representative interaction between a tactile sensor and the remote environment. Our objective is therefore twofold: (i) minimizing the number of actuators, and, (ii) optimizing the configuration of the (remaining) actuators to minimize the haptic rendering error. The algorithm is detailed below and summarized in Fig. 3.

\section{A. Tactile interaction and data-driven algorithm}

First, we record the target tactile interaction using the BioTac sensor. Let $s_{a}=\left[\begin{array}{llll}s_{1} & s_{2} & \ldots & s_{q}\end{array}\right]^{T} \in \mathbb{S}^{q} \in \mathbb{Z}^{q \times 20}$ be the series of tactile sensations the BioTac registers during our target interaction (e.g., for a one-minute-long tactile interaction recorded at $100 \mathrm{~Hz}, s_{a}$ is a $6000 \times 20$ matrix). Then, we run the recorded tactile interaction $s_{a}$ through our data-driven algorithm described in Sec. III. The algorithm leads to a series of $N$-tuples for the cutaneous device, as indicated in eq. (4). Let $\overline{\boldsymbol{m}}_{\boldsymbol{a}, \boldsymbol{N}}=\left[\begin{array}{llll}\overline{\boldsymbol{m}}_{\mathbf{1}} & \overline{\boldsymbol{m}}_{\mathbf{2}} & \ldots & \overline{\boldsymbol{m}}_{\boldsymbol{q}}\end{array}\right]^{T} \in \mathbb{M}^{q} \in \mathbb{R}^{q \times N}$ be the series of motor inputs computed by the algorithm for the target tactile interaction $s_{a}$ (e.g., for a one-minute-long tactile interaction recorded at $100 \mathrm{~Hz}$ and a $6-\mathrm{DoF}$ cutaneous device, $\boldsymbol{m}_{\boldsymbol{a}, \boldsymbol{6}}$ is a $6000 \times 6$ matrix). $\bar{m}_{a, N}$ represents the series of motor inputs that we would actuate on our original, non-optimized $N$-DoF cutaneous device to render the recorded BioTac sensations $s_{\boldsymbol{a}}$ on the operator's fingertip, as done in [6], [7].

\section{B. Principal Component Analysis}

Given the tactile interaction $s_{a}$ and the motor inputs $\overline{\boldsymbol{m}}_{\boldsymbol{a}, \boldsymbol{N}}$, we want to understand (i) if we can couple the behavior of some of the motors, and, if we can, (ii) how we can optimally couple them to minimize the haptic rendering error. Toward this objective, we run PCA on $\bar{m}_{\boldsymbol{a}, \boldsymbol{N}}$. PCA is used to emphasize variation and highlight strong patterns in a given dataset; it uses an orthogonal basis to transform a set of observations of (possibly correlated) variables into a set of values of linearly uncorrelated variables [16], [17]. The latter set of variables are called "principal components," and they are combinations of the original variables after a linear transformation. This transformation is such that the first principal component has the largest possible variance (i.e., it accounts for as much data variability as possible), and each succeeding component has, in turn, the highest variance possible given that it is orthogonal to the preceding components. Therefore, the number of components is upper bounded by the dimensionality of the given dataset ( $N$ in our case).

Let $\overline{\boldsymbol{m}}_{a, N}$ be our centered data matrix of motor inputs, such that the mean of each column is zero. Let $\boldsymbol{E}_{\boldsymbol{N}}$ be the $N \times N$ matrix containing all the principal components [17]. As mentioned above, we start by considering the principal components with the largest variance toward the ones with the smallest variance (i.e., the first column of $\boldsymbol{E}_{\boldsymbol{N}}$ contains the first principal component, while the $N^{\text {th }}$ column contains the last one). According to the number of principal components 


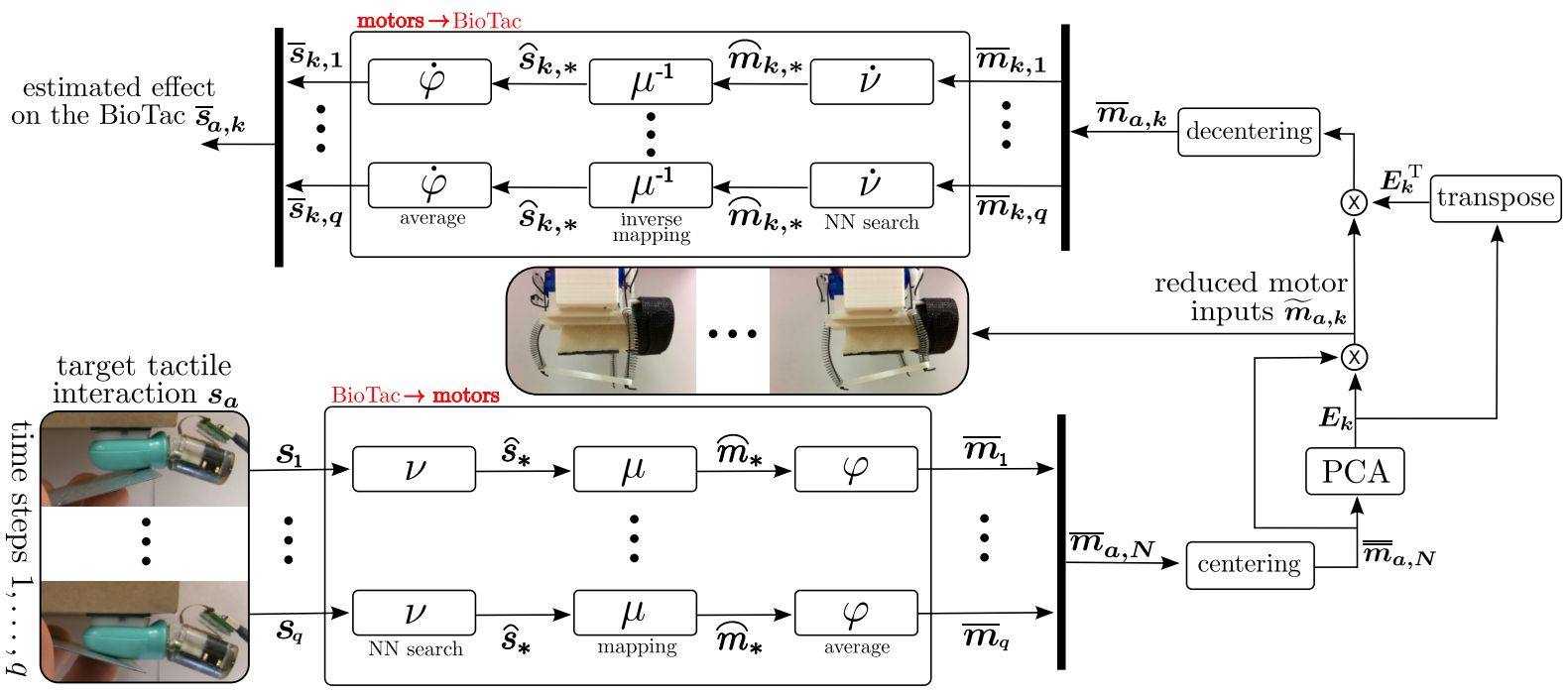

Fig. 3. Proposed algorithm. Starting from a target tactile interaction to render and a cutaneous device to optimize, it aims at finding the best number and configuration of motors a new cutaneous device needs to effectively render the target tactile sensations.

$k \leq N$ that we consider, we can calculate the new set of reduced motor inputs as

$$
\widetilde{\boldsymbol{m}}_{a, k}=\overline{\overline{\boldsymbol{m}}}_{a, N} \boldsymbol{E}_{\boldsymbol{k}} \in \mathbb{R}^{q \times k},
$$

where $\boldsymbol{E}_{\boldsymbol{k}} \in \mathbb{R}^{N \times k}$ is the matrix containing the first $k$ columns (i.e., principal components) of $\boldsymbol{E}_{N}$. The new matrix $\widetilde{\boldsymbol{m}}_{\boldsymbol{a}, \boldsymbol{k}}$ contains the motor inputs for the reduced set of $k$ DoF. For example, if we choose $k=1, \widetilde{\boldsymbol{m}}_{\boldsymbol{a}, \mathbf{1}} \in \mathbb{R}^{q}$ provides us with a single value for each tactile sensation registered by the BioTac, and $\boldsymbol{E}_{1}$ tells us how to optimally couple the inputs of the original $N$ motors into one. Similarly, if we choose $k=N$, $\widetilde{\boldsymbol{m}}_{\boldsymbol{a}, \boldsymbol{N}} \in \mathbb{R}^{q \times N}$ is equal to $\overline{\overline{\boldsymbol{m}}}_{\boldsymbol{a}, \boldsymbol{N}}$.

Moreover, by comparing $\widetilde{\boldsymbol{m}}_{\boldsymbol{a}, \boldsymbol{k}}$ and $\overline{\overline{\boldsymbol{m}}}_{\boldsymbol{a}, \boldsymbol{N}}$, we can also get a general idea of the rendering error expected from using $k<N$ rather than $N$. Toward this goal, let $\boldsymbol{\sigma}=\left[\begin{array}{llll}\sigma_{1} & \sigma_{2} & \ldots & \sigma_{N}\end{array}\right] \in$ $\mathbb{R}^{N}$ be the vector containing the variances of each principal component, i.e., the eigenvalues of the covariance matrix of $\overline{\overline{\boldsymbol{m}}}_{\boldsymbol{a}, \boldsymbol{N}}$. By dividing each variance by the total sum of the observed variances, we can evaluate how much each component explains the overall variability of the data,

$$
\boldsymbol{p}_{\boldsymbol{\sigma}}=\left[\begin{array}{llll}
\frac{\sigma_{1}}{\sum_{i=1}^{N} \sigma_{i}} & \frac{\sigma_{2}}{\sum_{i=1}^{N} \sigma_{i}} & \cdots & \frac{\sigma_{N}}{\sum_{i=1}^{N} \sigma_{i}}
\end{array}\right] .
$$

Naturally, we expect this value to significantly affect the final rendering error.

As mentioned above, considering the kinematics of the cutaneous device employed, the PCA coefficients in $\boldsymbol{E}_{\boldsymbol{k}}$ provide us with information on how to optimally couple the $N$ DoF of the original device into the $k$ most important ones that we want to choose. For example, if our target interaction contains solely the "pressure" EP [13], we may not need more than one degree of freedom to properly render it. If the considered cutaneous device has more degrees of freedom than that (i.e., $N>1$ ), and if it is able to render the recorded "pressure" interaction, the PCA coefficients in $\boldsymbol{E}_{\boldsymbol{1}}$ will show us how to couple, in an optimal fashion, the cutaneous device's $N$ DoF into a single one. From this result, we could decide to build a new cutaneous device composed of only one motor, which moves the platform along the first principal component indicated by our analysis.

\section{Choosing the number of principal components}

Choosing the right number of principal components is challenging: we need to compromise between a complex actuation system and a good rendering performance. The explained variance vector $\boldsymbol{p}_{\boldsymbol{\sigma}}$ already gives us a good idea of how well each principal component can describe the variability of our data. However, it would be beneficial to quantitatively evaluate the tactile rendering error in terms of the difference between the target and rendered tactile sensations.

Toward this objective, we can reconstruct [17] the $N$ motor inputs data using the reduced set of $k=1, \ldots, N$ components,

$$
\overline{\boldsymbol{m}}_{\boldsymbol{a}, \boldsymbol{k}}=\widetilde{\boldsymbol{m}}_{\boldsymbol{a}, \boldsymbol{k}} \boldsymbol{E}_{\boldsymbol{k}}^{\mathrm{T}}+\operatorname{mean}\left(\overline{\boldsymbol{m}}_{\boldsymbol{a}, \boldsymbol{N}}\right), \quad \text { for } k=1, \ldots, N
$$

where the "mean" factor is a $1 \times k$ vector composed of the means of the first $k$ columns of $\overline{\boldsymbol{m}}_{\boldsymbol{a}, \boldsymbol{N}}$. Vectors $\overline{\boldsymbol{m}}_{\boldsymbol{a}, \boldsymbol{k}}=$ $\left[\begin{array}{llll}\overline{\boldsymbol{m}}_{\boldsymbol{k}, \mathbf{1}} & \overline{\boldsymbol{m}}_{\boldsymbol{k}, \mathbf{2}} & \ldots & \overline{\boldsymbol{m}}_{\boldsymbol{k}, \boldsymbol{q}}\end{array}\right]^{T} \in \mathbb{M}_{k}^{q} \in \mathbb{R}^{q \times N}$ and $\overline{\boldsymbol{m}}_{\boldsymbol{a}, \boldsymbol{N}}$ store the reconstructed and original motor inputs for our target interaction, respectively. Note that both $\overline{\boldsymbol{m}}_{\boldsymbol{a}, \boldsymbol{k}}$ and $\overline{\boldsymbol{m}}_{\boldsymbol{a}, \boldsymbol{N}}$ have the same dimensions, i.e., $q \times N$. Of course, as $k$ gets smaller, $\overline{\boldsymbol{m}}_{\boldsymbol{a}, \boldsymbol{k}}$ loses important information toward a good reconstruction of the original motor inputs $\overline{\boldsymbol{m}}_{a, N}$.

In order to evaluate the tactile rendering error, we now need to understand the effect of using the reconstructed motor inputs $\overline{\boldsymbol{m}}_{\boldsymbol{a}, \boldsymbol{k}}$ vs. the original $\overline{\boldsymbol{m}}_{\boldsymbol{a}, \boldsymbol{N}}$. A good approach is to place the BioTac inside the cutaneous device again, as in Figs. 1b and $2 \mathrm{~b}$, and play $\overline{\boldsymbol{m}}_{\boldsymbol{a}, \boldsymbol{k}}$ for $k=1, \ldots, N$ through the motors. Then, the data recorded by the BioTac during this process can be compared to (i) the target tactile sensations $s_{a}$ and to (ii) the tactile sensations registered by the BioTac when actuating $\overline{\boldsymbol{m}}_{\boldsymbol{a}, \boldsymbol{N}}$. This process would enable us to directly evaluate the difference between the tactile interaction rendered when using the reduced $k$ DoF of the device vs. the original target tactile sensation, and vs. the tactile interaction rendered when using all $N$ DoF of the device. 
Although effective, the above-mentioned approach may be very time consuming, as it needs to run the target tactile interaction through the considered cutaneous device $N$ times. An alternative approach is to re-use the data gathered during the first data collection of Sec. III-A to estimate the outcome of these new tactile interactions. This approach not only saves a significant amount of time, but it also reduces the need to physically have the original $N$-DoF cutaneous device used for the data collection. In fact, all we need for the proposed optimization algorithm is the initial data collection look-up table and the target tactile interaction. The implications of this fact will be discussed in detail in Sec. VI.

Function $\mu(\cdot)$ of eq. (1) defines a look-up table linking the BioTac's electrode and DC pressure readings $s_{*}$ to the corresponding tested motor inputs $\boldsymbol{m}_{*}$. If each $N$-tuple $\overline{\boldsymbol{m}}_{\boldsymbol{k}}$ in $\overline{\boldsymbol{m}}_{\boldsymbol{a}, \boldsymbol{k}}$ were in the set of motor $N$-tuples tested during the first data collection, i.e., if all $\overline{\boldsymbol{m}}_{\boldsymbol{k}} \in \mathbb{M}_{*}$, we could simply invert $\mu(\cdot)$ to retrieve each corresponding BioTac sensation, i.e., $\mu^{-1}\left(\overline{\boldsymbol{m}}_{\boldsymbol{k}}\right)=s_{*}$. However, this is usually not the case, i.e., $\mathbb{M}_{*}^{q} \neq \mathbb{M}_{k}^{q}$. We address this problem by looking for the $n$ motor inputs in our domain $\mathbb{M}_{*}$ closest to each reconstructed input $\overline{\boldsymbol{m}}_{\boldsymbol{k}}$, thus defining

$$
\dot{\nu}: \mathbb{M}_{k} \rightarrow \mathbb{M}_{*}^{n}, \quad \dot{\nu}\left(\overline{\boldsymbol{m}}_{\boldsymbol{k}}\right)=\left[\begin{array}{c}
\boldsymbol{m}_{\boldsymbol{k}, *, \mathbf{1}} \\
\vdots \\
\boldsymbol{m}_{\boldsymbol{k}, *, n}
\end{array}\right]=\widehat{\boldsymbol{m}}_{\boldsymbol{k}, *},
$$

as the function that maps a reconstructed motor $N$-tuple $\overline{\boldsymbol{m}}_{\boldsymbol{k}} \in$ $\mathbb{M}_{k}$, generated from eq. (7), to the $n$ closest ones in $\mathbb{M}_{*}$. As before, also here we use $n=8$.

We can now map the $n$ points retrieved by $\dot{\nu}(\cdot)$ to their corresponding tactile sensations registered during the data collection,

$$
\begin{aligned}
& \mu^{-1}: \mathbb{M}_{*}^{n} \rightarrow \mathbb{S}_{*}^{n}, \\
& \mu^{-1}\left(\widehat{\boldsymbol{m}}_{\boldsymbol{k}, *}\right)=\mu^{-1}\left(\left[\begin{array}{c}
\boldsymbol{m}_{\boldsymbol{k}, *, \mathbf{1}} \\
\vdots \\
\boldsymbol{m}_{\boldsymbol{k}, *, \boldsymbol{n}}
\end{array}\right]\right)=\left[\begin{array}{c}
\boldsymbol{s}_{\boldsymbol{k}, *, \mathbf{1}} \\
\vdots \\
\boldsymbol{s}_{\boldsymbol{k}, *, \boldsymbol{n}}
\end{array}\right]=\hat{s}_{\boldsymbol{k}, *},
\end{aligned}
$$

Finally, we average $\hat{s}_{\boldsymbol{k}, *} \in \mathbb{S}_{*}^{n}$ to a single tactile sensation as

$$
\dot{\varphi}: \mathbb{S}_{k, *}^{n} \rightarrow \mathbb{S}, \quad \dot{\varphi}\left(\hat{s}_{k, *}\right)=\varphi\left(\left[\begin{array}{c}
s_{k, *, 1} \\
\vdots \\
s_{k, *, n}
\end{array}\right]\right)=\bar{s}_{k},
$$

considering a simple inverse squared distance mean that weights tactile sensations according to the inverse squared distance between the corresponding motor input in $\mathbb{M}_{*}$ and the reconstructed input $\overline{\boldsymbol{m}}_{\boldsymbol{k}}$ (as in eq. (4) and [6]). Vector $\overline{\boldsymbol{s}}_{\boldsymbol{k}} \in \mathbb{S}$ is our final estimated tactile sensation caused by the actuation of $\overline{\boldsymbol{m}}_{\boldsymbol{k}}$.

Let $\bar{s}_{a, k}=\left[\begin{array}{llll}\bar{s}_{k, 1} & \bar{s}_{k, 2} & \ldots & \bar{s}_{k, q}\end{array}\right]^{T} \in \mathbb{S}^{q}$ be the series of tactile sensations caused by $\overline{\boldsymbol{m}}_{\boldsymbol{a}, \boldsymbol{k}}$, estimated following the described procedure. Now, we can finally evaluate the estimated tactile rendering error by comparing $\bar{s}_{a, k}$ to the original target tactile sensation $s_{a}$,

$$
e_{s, k}=\left\|\bar{s}_{a, k}-s_{a}\right\|
$$

The root mean square $\bar{e}_{s, k}$ of $\boldsymbol{e}_{\boldsymbol{s}, \boldsymbol{k}}$ gives us an absolute measure of the tactile rendering error. Moreover, comparing $\bar{s}_{a, k}$ to $\overline{\boldsymbol{s}}_{\boldsymbol{a}, \boldsymbol{N}}$ gives us information about how using $k<N$ principal components affects the tactile rendering. Let $e_{s, N}=\| \bar{s}_{a, N}-$ $s_{a} \|$ be the tactile rendering error when using the original
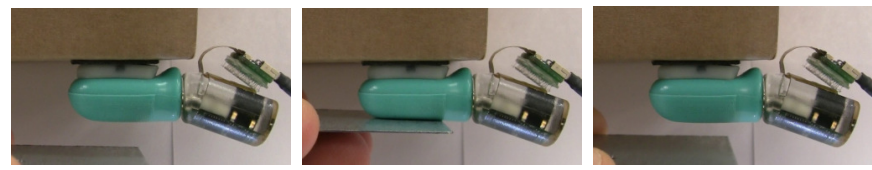

(a) Flat interaction
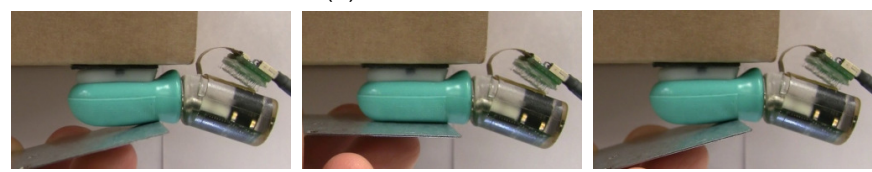

(b) Back-flat-back interaction
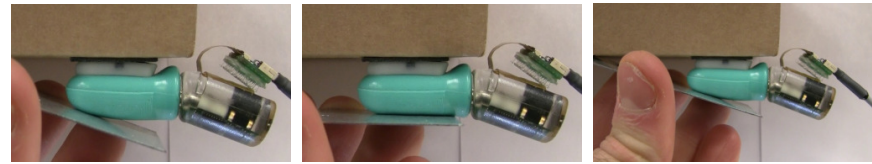

(c) Tip-flat-tip interaction
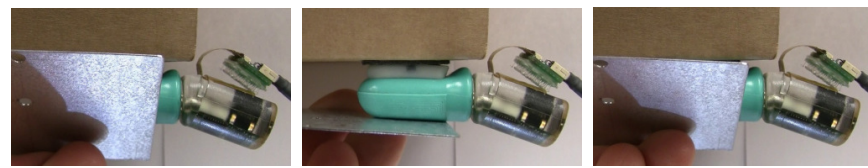

(d) Left-flat-left interaction
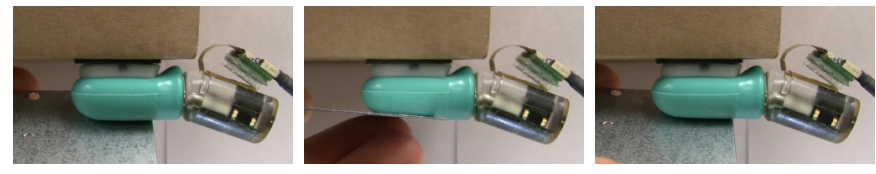

(e) Right-flat-right interaction
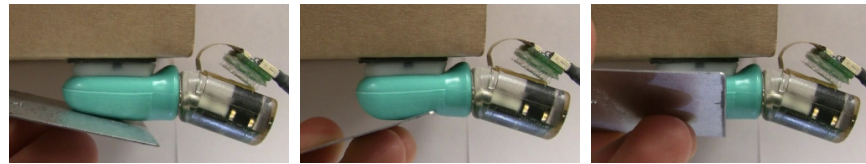

(f) Complex interaction (a combination of the others)

Fig. 4. Recorded interactions. Tactile data was recorded during six different interactions between a BioTac sensor and a flat surface. Adapted from [6].

mapping algorithm (i.e., when using all the available $N$ DoF), and let $\bar{e}_{s, N}$ be its root mean square. We can now define as

$$
\bar{e}_{\%, k}=100 \frac{\bar{e}_{s, k}-\bar{e}_{s, N}}{\bar{e}_{s, N}}
$$

the percentage error of $\bar{e}_{s, k}$ with respect to $\bar{e}_{s, N}$. Since $\bar{e}_{s, N}$ is the estimated error when using the original $N$-DoF device, it represents the best performance we can achieve with the considered system. For this reason, it makes sense to evaluate the new error $\bar{e}_{s, k}$ with respect to $\bar{e}_{s, N}$. In general, we expect $\bar{e}_{\%, k}$ (and $\bar{e}_{s, k}$ ) to decrease as $k$ increases, up to the point at which no further improvements are possible. Of course, we also expect a lower rendering error to yield tactile sensations that feel more realistic to a human user, as shown in [6].

\section{EXPERIMENTAL EVALUATION}

We validate this approach for the two devices of Sec. II.

\section{A. Set of target tactile experiences}

We considered as our target tactile experiences the same tactile interactions used in [6]. In that paper, we recorded tactile data during six different interactions between a stationary BioTac sensor and a flat metal surface that was moved by hand to touch the BioTac's fingertip in different ways. The experimental setup is shown in Fig. 4, and the supplementary 
video shows all the interactions. The first interaction, called flat in Fig. 4a, consists of making contact with the finger pulp with the surface parallel to the sensor's nail. We expect to need only a 1-DoF cutaneous device to render this interaction, requiring the device's mobile platform to move toward and away from the BioTac with the surface parallel to the sensor's nail. The second interaction, called back-flat-back in Fig. 4b, consists of making contact with the proximal part of the BioTac, then moving toward the finger pulp and returning to the back part of the finger. The third one, called tip-flat-tip in Fig. 4c, consists of making contact with the distal part of the BioTac, then moving toward the finger pulp and returning to the tip. The fourth one, called left-flat-left in Fig. 4d, consists of making contact on the left lateral side of the BioTac, then moving toward the finger pulp and returning to the left side. The fifth one, called right-flat-right in Fig. 4e, performs the same sequence of interactions on the right lateral side of the BioTac. We expect to need only a 2-DoF cutaneous device to render any of these four interactions, as they add a proximal, distal, or lateral tactile movement to the flat interaction. The last one, called complex in Fig. 4f, is a combination of the other five motions. For this reason, we expect it to need a 3-DoF cutaneous device to be properly rendered. Each interaction was repeated three times within its recording. The interactions lasted 210 seconds in total, with 21057 tactile sensations recorded by the BioTac. These six target tactile experiences were chosen to include interactions (i) as general as possible, (ii) able to be correctly rendered with both our 3-DoF and 6-DoF cutaneous devices, (iii) requiring different (and known) numbers and configurations of the actuators. We used these target experiences as ground truth, to test whether our approach could accurately predict the number of DoF needed to display a given tactile interaction. In fact, the algorithm has no prior knowledge of the nature of such experiences; the only input it receives is the stream of target tactile interactions $\boldsymbol{s}_{\boldsymbol{a}}$ and the look-up table.

\section{B. Results for the 3-DoF cutaneous device}

The estimated rendering error $\bar{e}_{s, k}$ for the 3-DoF device, averaged across interactions, is 167.9, 119.2, and 105.4 bits when using $k=1,2,3$ principal components, respectively (not shown in the figures). Figure 5 shows the percentage error $\bar{e}_{\%, k}$ of $\bar{e}_{s, k}$ with respect to $\bar{e}_{s, 3}$. This error, averaged across interactions, is $55.3 \%$ and $13.4 \%$ when using $k=1,2$ principal components, respectively. The lowest value of $k$ that produces an error similar to $k=N$ varies by interaction. The lower the $k$, the fewer actuators our new optimized device will need.

In order to validate our estimation of the rendering errors $\bar{e}_{s, k}$, we placed the BioTac inside the cutaneous device, as done for data collection (see Fig. 1b). Then, we sent the data from the six tactile interactions through the mapping algorithm of Sec. III-B and drove the cutaneous device to the resulting motor angle triplets over time while recording what the BioTac sensed. Averaged across sensing channels and over time, we calculated the mean difference between the estimated tactile sensations $\bar{s}_{\boldsymbol{a}, 3}$ and the ones created by the cutaneous device on the BioTac. Results showed an average error of $56.9 \pm 19.2$ bits, which is about $1.3 \%$ of the full 12-bit scale the BioTac can reach $(0-4095)$.

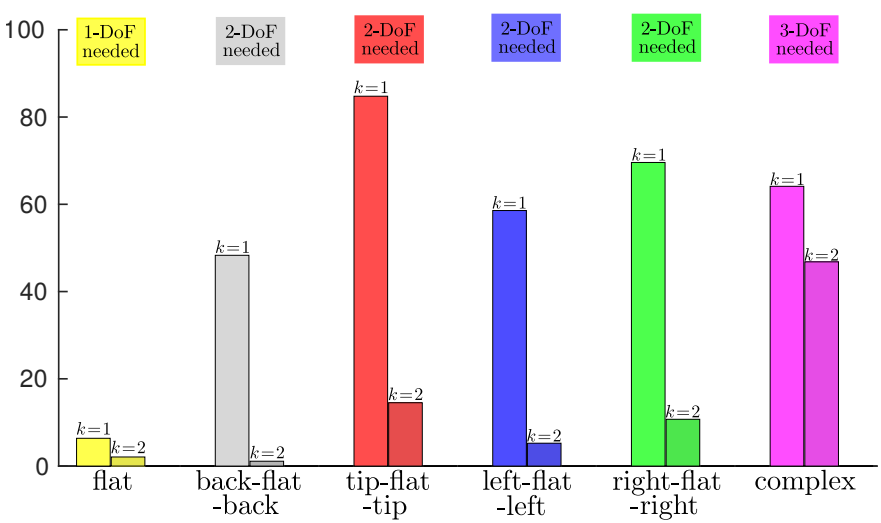

Fig. 5. Results for the 3-DoF cutaneous device: percentage error $\bar{e}_{\%, k}$ for the six tactile interactions using $\mathrm{k}=1$ and $\mathrm{k}=2$ principal components.

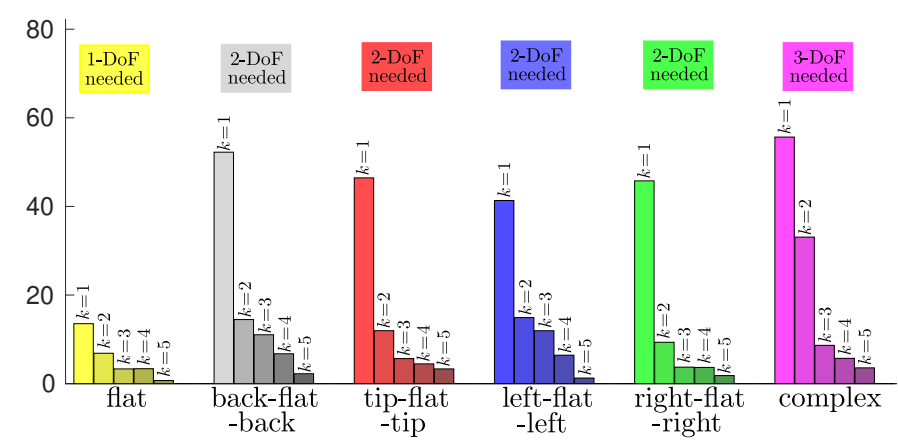

Fig. 6. Results for the 6-DoF cutaneous device: percentage error $\bar{e}_{\%, k}$ for the six tactile interactions using from $\mathrm{k}=1$ to $\mathrm{k}=5$ principal components.

\section{Results for the 6-DoF cutaneous device}

The estimated rendering error $\bar{e}_{s, k}$ for the 6-DoF device, averaged across interactions, is $219.9,175.0,163.9,159.8$, 155.1, and 151.7 bits when using $k=1, \ldots, 6$ principal components, respectively (not shown in the figures). Figure 6 shows the percentage error $\bar{e}_{\%, k}$ of $\bar{e}_{s, k}$ with respect to $\bar{e}_{s, 6}$. This error, averaged across interactions, is $42.5 \%, 15.1 \%, 7.4 \%$, $5.1 \%$, and $2.2 \%$ when using $k=1, \ldots, 5$ principal components. Again, the optimal value of $k$ varies across interactions.

\section{Discussion, CONClusions, And Future Work}

The paper introduced a novel task-driven approach for the design optimization of cutaneous haptic devices, an approach that can serve as a starting point for similar dataand statistically-inspired design optimization techniques. We validated our approach using a BioTac tactile sensor and custom 3-DoF and 6-DoF fingertip cutaneous devices, considering six representative tactile interactions.

Section V-B reports the results for our 3-DoF cutaneous device. The resulting rendering errors prove the effectiveness of our approach in correctly understanding the nature of the considered target interactions. As expected, the flat interaction is well rendered even when using only the first principal component, and no significant improvement is achieved by adding more DoF. If this was our target interaction, we could now design a new cutaneous device with only one motor. Moreover, the coefficients of the first principal component, $\boldsymbol{E}_{\mathbf{1}, \text { flat }}=[0.58,0.60,0.56]^{T}$, tell us how each cable should move relative to this single motor; Fig. 7a shows how this principal component moves the platform. A possible design for 
this optimized 1-DoF cutaneous device is shown in Fig. 7b. The diameters of the three pulleys attached to the servo motor, which control the length of the cables, are multiples of $\boldsymbol{E}_{1, \text { flat }}$. The new device is more compact than the original 3-DoF version and weighs $54 \%$ less. This $1-$ DoF design can be optimized for any target interaction that is well-rendered using only the first principal component, simply by using the proposed method to find the coefficients of the first principal component and adjusting the pulley diameters accordingly.

On the other hand, as expected, the tip-flat-tip interaction requires at least two principal components to be rendered correctly by our device. This means that we could design a new cutaneous device with only two motors, and the coefficients of the first two principal components can tell us how each cable should move relative to each motor. In our case, $\boldsymbol{E}_{\mathbf{2}, \text { tip }}=[0.45,0.65,0.61 ; 0.89,-0.34,-0.30]^{T}$ (illustrated in Fig. 8a). A possible design for this optimized 2 -DoF cutaneous device is shown in Fig. 8b; as before, the diameters of the six pulleys attached to the two servo motors are multiples of $\boldsymbol{E}_{2, t i p}$. The new device is again more compact than the original $3-\mathrm{DoF}$ version and weighs $15 \%$ less. A similar approach can be used for the back-flat-back, left-flat-left, and right-flat-right interactions. They all require two principal components to be effectively rendered.

Finally, the complex interaction requires all three principal components. In this case, it is not possible to couple the behavior of any of the three motors without losing significant parts of the interaction.

The principal components also provide insight into how the redesigned device should roughly behave. We see in the first principal component of the flat interaction that all three coefficients are roughly the same, meaning all three cables should always extend or retract together. In fact, by doing so, the mobile platform can move similar to how the metal surface moves in the flat interaction of Fig. 4a. For the tip-flattip interaction, we see that the second and third coefficients are similar for both principal components, meaning the two cables on the proximal part of the device (the ones close to the fabric strap) should roughly move together. The insight provided by the principal components may be particularly helpful when looking at higher-degree-of-freedom systems, when it is no longer feasible to use a network of pulleys and gears to link every cable to every motor. Lighter devices with similar rendering capabilities may be obtained by finding subsets of the principal component coefficients that are similar, and coupling the corresponding set of cables by having one motor directly control only those cables. There are indeed no particular restrictions on how to actuate these couplings; the presented devices are only two possible designs.

Section V-B also evaluates the quality of our rendering error estimation, which shows an error of about $1.3 \%$ of the full BioTac's sensing range for $\bar{e}_{s, N}$. We expect this estimation error to decrease as more points are added to the look-up table and as these points more closely match the target tactile interactions. For this reason, this error can also be seen as a direct evaluation of the data collection process for the considered interactions.

Section V-C reports the results for our 6-DoF cutaneous device. Similarly to what we have seen for the 3-DoF device,

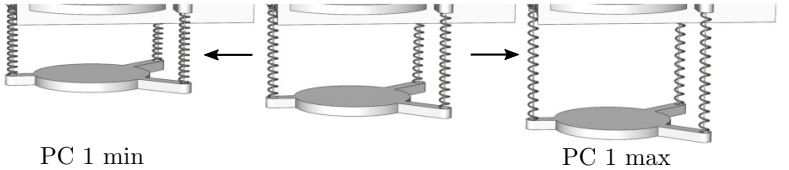

(a) First principal component motion for the flat interaction rendered by the 3-DoF device.
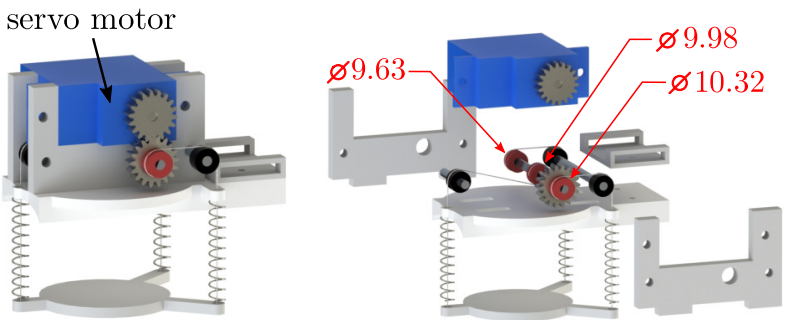

(b) New 1-DoF fingertip device optimized for the flat interaction.

Fig. 7. An example of how our custom 3-DoF cutaneous device could be re-designed for the flat interaction using the proposed optimization approach. Radii are in millimeters.

the results show the effectiveness of our approach in correctly understanding the nature of the considered target interactions. The flat interaction is correctly rendered even when using only the first principal component. On the other hand, interactions tip-flat-tip, back-flat-back, left-flat-left, and right-flat-right need at least two principal components. Finally, the complex interaction requires at least three principal components to be effectively rendered. Since the flat metal surface roughly moves in a three-dimensional subspace during all target interactions, using more than three principal components does not add much information to the rendered tactile interaction. Interestingly, the overall average error is somewhat higher when using the 6-DoF device than the 3-DoF device. We can explain this behavior by remembering the different number of points tested during data collection, only 4033 for the 6-DoF device vs. 166375 for the 3-DoF device.

These results preliminarily show the effectiveness and viability of using the proposed approach to optimize the design of fingertip cutaneous devices. The algorithm starts from a target tactile interaction to render and a cutaneous device to optimize. Of course, as the algorithm analyzes the couplings between the motor motions of the original cutaneous device, the latter should be able to correctly render the target tactile interaction. In fact, the performance of the original cutaneous device can be considered as the upper bound on performance for any new cutaneous device re-designed using our algorithm. For this reason, it is important to start the optimization process from a cutaneous device with high actuation capabilities (e.g., high DoF, large workspace, high applicable forces). At the same time, it is also important to run a proper data collection process, covering in detail as much workspace as possible. However, this is not always easy. For example, covering the entire workspace of our 6-DoF device may take a very long time (e.g., around 74 days if we consider 20 steps per dimension). For this reason, it is important to develop clever ways of building the lookup tables, i.e., discarding areas of the workspace that are not interesting (e.g., where the end-effector does not contact the finger) while focusing on those configurations bringing the most salient information to the tactile interaction. We plan to address this problem in the near future. Moreover, since 


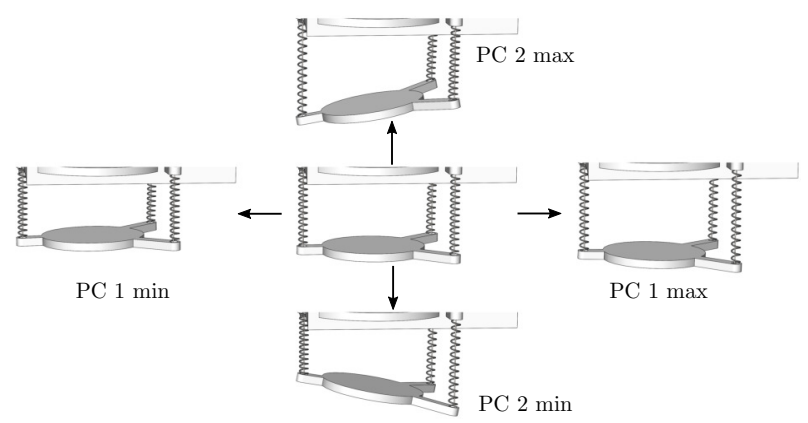

(a) Motion of the first and second principal components for the tip-flat-tip interaction rendered by the 3-DoF device.

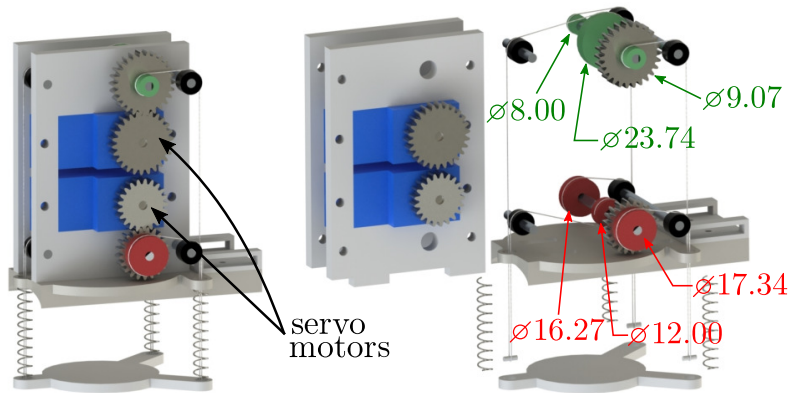

(b) New 2-DoF fingertip device optimized for the tip-flat-tip interaction.

Fig. 8. A second example of how our custom 3-DoF cutaneous device could be re-designed using the proposed optimization approach. Radii are in millimeters Red pulleys are designed using information from the first PC, while green pulleys are designed using information from the second PC.

the look-up table is the only data we need from the original cutaneous device, it is not necessary to physically have the device. In fact, any researcher with the look-up tables of our 3-DoF and 6-DoF cutaneous devices can run the optimization algorithm for their custom target tactile interactions and derive a new optimized device. Readers can find the data collection files for our 3-DoF device as supplemental material. They contain the commanded motor angle triplets for the cutaneous device and the corresponding tactile sensations registered by the BioTac sensor. Researchers can use these data to create a custom optimized device for their own target tactile interactions. The only requirement is to consider tactile interactions that can be adequately rendered by the original 3-DoF device.

This paper serves as a starting point for optimizing the design of cutaneous interfaces using data from target interactions, and we plan to expand these methods in the future. Specifically, we intend to run a human subject study to compare the tactile rendering performance of the original devices to that of the optimized ones. This study will quantitatively assess how the rendering error affects the performance of representative tasks, to elucidate the level of error that can be tolerated when reducing a device's DoF. Of course, we expect lower rendering error to yield more realistic tactile sensations, as demonstrated in our previous work [6]. That article dealt with the same 3-DoF device and the same six fingertip interactions presented here. In one experiment, we artificially constrained the 3-DoF device to operate with only one degree of freedom during the human-subject study, in addition to operating normally. When controlling it as a $1-\mathrm{DoF}$ device, we commanded the three motors to the same target angle (i.e., $m_{1}=m_{2}=m_{3}$ ). Although this synergy was chosen intuitively, it is interesting to note that it is almost identical to the first principal component discovered by our algorithm in this work. Human subjects then watched videos of the different recorded interactions while feeling either the 1-DoF or the 3-DoF version of the device render the sensations. Our ten participants rated the 1-DoF rendering of the flat interaction to be no different from the 3-DoF rendering of this same interaction, giving both approximately a 9.3 out of 10 , where 10 means that the sensations corresponded very well to what the subject expected. In addition to carrying out this human subject study, we will also investigate how to extract additional useful information from the raw tactile sensor data, such as the suitability of the original cutaneous device. Finally, we also plan to evaluate the performance of our approach with a broader set of tactile interactions. In fact, the proposed approach is especially useful for complex tactile interactions, where one cannot intuitively infer the optimal device design.

\section{REFERENCES}

[1] S. B. Schorr and A. Okamura, "Three-dimensional skin deformation as force substitution: Wearable device design and performance during haptic exploration of virtual environments," IEEE Trans. Haptics, 2017.

[2] M. Maisto, C. Pacchierotti, F. Chinello, G. Salvietti, A. De Luca, and D. Prattichizzo, "Evaluation of wearable haptic systems for the fingers in augmented reality applications," IEEE Trans. Haptics, 2017.

[3] C. Pacchierotti, L. Meli, F. Chinello, M. Malvezzi, and D. Prattichizzo, "Cutaneous haptic feedback to ensure the stability of robotic teleoperation systems," Int. J. Robot. Res., vol. 34, no. 14, pp. 1773-1787, 2015.

[4] H. Benko, C. Holz, M. Sinclair, and E. Ofek, "NormalTouch and TextureTouch: High-fidelity 3D haptic shape rendering on handheld virtual reality controllers," in Proc. Symp. on User Interface Software and Technology, 2016, pp. 717-728.

[5] C. Pacchierotti, S. Sinclair, M. Solazzi, A. Frisoli, V. Hayward, and D. Prattichizzo, "Wearable haptic systems for the fingertip and the hand: taxonomy, review, and perspectives," IEEE Trans. Haptics, 2017.

[6] C. Pacchierotti, D. Prattichizzo, and K. J. Kuchenbecker, "Displaying sensed tactile cues with a fingertip haptic device," IEEE Trans. Haptics, vol. 8, no. 4, pp. 384-396, 2015.

[7] — "Cutaneous feedback of fingertip deformation and vibration for palpation in robotic surgery," IEEE Trans. Biomedical Engineering, vol. 63 , no. 2, pp. 278-287, 2016.

[8] N. Wettels and G. E. Loeb, "Haptic feature extraction from a biomimetic tactile sensor: force, contact location and curvature," in Proc. IEEE International Conf. on Robotics and Biomimetics, 2011, pp. 2471-2478.

[9] J. A. Fishel and G. E. Loeb, "Sensing tactile microvibrations with the BioTac-comparison with human sensitivity," in Proc. IEEE International Conf. on Robotics and Biomimetics, 2012, pp. 1122-1127.

[10] E. Young and K. J. Kuchenbecker, "Design of a parallel continuum manipulator for 6-DoF fingertip haptic display," in Proc. IEEE World Haptics, 2017.

[11] M. C. Jimenez and J. A. Fishel, "Evaluation of force, vibration and thermal tactile feedback in prosthetic limbs," in Proc. IEEE Haptics Symposium, 2014, pp. 437-441.

[12] J. D. Brown, M. Ibrahim, E. D. Z. Chase, C. Pacchierotti, and K. J. Kuchenbecker, "Data-driven comparison of four cutaneous displays for pinching palpation in robotic surgery," in Proc. IEEE Haptics Symposium, 2016, pp. 147-154.

[13] S. J. Lederman and R. L. Klatzky, "Hand movements: A window into haptic object recognition," Cogn. Psychol., vol. 19, no. 3, pp. 342-368, 1987.

[14] S. Scheggi, L. Meli, C. Pacchierotti, and D. Prattichizzo, "Touch the virtual reality: using the Leap Motion controller for hand tracking and wearable tactile devices for immersive haptic rendering," in Proc. ACM SIGGRAPH, 2015.

[15] S. B. Schorr and A. M. Okamura, "Fingertip tactile devices for virtual object manipulation and exploration," in Proc. CHI, 2017, pp. 3115-3119.

[16] P. M. Jain and V. Shandliya, "A survey paper on comparative study between principal component analysis (PCA) and exploratory factor analysis (EFA)," Intl. J. Management, IT and Engineering, vol. 3, no. 6, p. $415,2013$.

[17] I. Jolliffe, Principal Component Analysis. Wiley Online Library, 2002. 